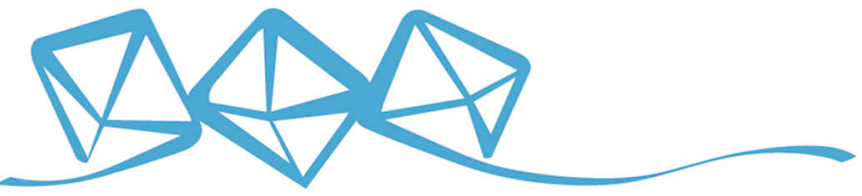 COMMUNICATIONS MATERIALS
}

ARTICLE

https://doi.org/10.1038/s43246-020-00072-4 OPEN

\section{Piezoelectricity in perovskite-type pseudo-cubic ferroelectrics by partial ordering of off-centered cations}

\author{
Yoshihiro Kuroiwa (i] ${ }^{1 凶}$, Sangwook Kim (10) ${ }^{1}$, Ichiro Fujii (1) ${ }^{2}$, Shintaro Ueno ${ }^{2}$, Yuki Nakahira ${ }^{3}$ \\ Chikako Moriyoshi id ${ }^{1}$, Yukio Sato $\mathbb{1}^{4} \&$ Satoshi Wada ${ }^{2}$
}

A large piezoelectric response in ferroelectric ceramics is typically associated with extrinsic contributions from ferroelectric domain structures. However, such domain structures cannot be expected in systems with pseudo-cubic symmetry. In this study, we determine the mechanism of significant piezoelectricity and ferroelectricity in $0.3 \mathrm{BaTiO}-0.1 \mathrm{Bi}\left(\mathrm{Mg}_{1 / 2} \mathrm{Ti}_{1 / 2}\right)$ $\mathrm{O}_{3}-0.6 \mathrm{BiFeO}_{3}$ ceramic with a perovskite-type pseudo-cubic symmetry. Synchrotron radiation $\mathrm{X}$-ray diffraction reveals that the $\mathrm{Bi}$ ions in this ceramic essentially prefer to be off-centered at six sites by approximately $0.4 \AA$, in the cubic $<100>$ directions. A phase transition occurs at $T_{C} \sim 725 \mathrm{~K}$. However, the crystal seems to present a cubic symmetry even at room temperature. The large piezoelectric response is caused by the combinational partial ordering of the off-centered $\mathrm{Bi}$ ions, adapted to any direction of the applied electric field to the ceramic grains. The proposed mechanism for the emergence of a high polarization in the above system will enable designing novel Pb-free ceramics by controlling the fluctuated and offcentered ions under an applied electric field.

\footnotetext{
${ }^{1}$ Graduate School of Advanced Science and Engineering, Hiroshima University, Higashihiroshima, Hiroshima $739-8526$, Japan. ${ }^{2}$ Graduate Faculty of Interdisciplinary Research, University of Yamanashi, Kofu, Yamanashi 400-8510, Japan. ${ }^{3}$ Department of Physical Science, Graduate School of Science, Hiroshima University, Higashihiroshima, Hiroshima 739-8526, Japan. ${ }^{4}$ Department of Materials Science and Engineering, Graduate School of Engineering, Kyushu University, Fukuoka 819-0395, Japan. ${ }^{凶}$ email: kuroiwa@sci.hiroshima-u.ac.jp
} 
$\mathrm{T}$ hus far, $\mathrm{Pb}$-based ferroelectric oxides with perovskite-type structures, as typified by $\mathrm{Pb}\left(\mathrm{Zr}_{1-x} \mathrm{Ti}_{x}\right) \mathrm{O}_{3}(\mathrm{PZT})$, have been mainly used as piezoelectric materials in various applications. It is well known that PZT ceramics exhibit excellent electric properties at morphotropic phase-boundary compositions $(x$ $\sim 0.5$ ), at which the coexistence of several structures, such as rhombohedral, tetragonal, and other structures (e.g., monoclinic structures), with different spontaneous lattice distortions is suggested $^{1-4}$. Recently, in relation to hazardous waste and problematic materials, the development of $\mathrm{Pb}$-free piezoelectric materials is rapidly progressing ${ }^{5-7}$. Particularly, it is known that Bi-based ceramics, such as $\mathrm{BiFeO}_{3}(\mathrm{BF})$ - and $\left(\mathrm{Bi}_{0.5} \mathrm{Na}_{0.5}\right) \mathrm{TiO}_{3}$ (BNT)-based perovskite-type solid solutions, have significant potential for displaying excellent piezoelectricity ${ }^{8-11}$. Our group has been searching for novel $\mathrm{Pb}$-free piezoelectric materials among solid solutions of Bi-based relaxor and standard ferroelectrics. We have deduced that solid solutions of $\mathrm{BaTiO}_{3}-\mathrm{Bi}$ $\left(\mathrm{Mg}_{1 / 2} \mathrm{Ti}_{1 / 2}\right) \mathrm{O}_{3}$ (BT-BMT) and BF (represented as BT-BMT-BF) are promising alternatives to $\mathrm{Pb}$-based materials, owing to their relatively large piezoelectric response and high Curie temperature $\left(T_{\mathrm{C}}\right)^{12}$.

Early structural studies via X-ray diffraction (XRD) suggested a cubic symmetry for BT-BMT-BF ceramics ${ }^{13,14}$. Hence, the unexpectedly large piezoelectric strain electrically observed in strain-electric-field $(S-E)$ curves, and the significant ferroelectricity presented in polarization-electric field $(P-E)$ hysteresis loops cannot be explained by the crystal symmetry. These unforeseen electric responses are not only unique to BT-BMT-BF ceramics, but have also been reported for other Bibased piezoelectric systems possessing a pseudocubic symmetry ${ }^{15-17}$. Thus, the reason for the observed high piezoelectricity and ferroelectricity is unclear.

Various models have been proposed in relation to the properties of ferroelectricity, piezoelectricity, and/or dielectric properties in other Bi-based materials with pseudocubic symmetry ${ }^{18-20}$. Recently, it was reported that the high piezoelectric response exhibited in BNT-BT single crystal with pseudocubic symmetry is due to improper ferroelectric distortion caused by in-phase or antiphase oxygen octahedral tilting ${ }^{21}$. Besides, detailed studies of local structures in Bi-based solid solutions were reported. It was suggested that the $\mathrm{Bi}$ displacements change as a function of temperature, and that a phase transition could be associated with the ferroelectric response ${ }^{22}$. Moreover, it was reported that the high piezoelectric response appeared by the local distortion in pseudocubic symmetry under an electric field ${ }^{23}$. Therefore, the structural characteristics related to the $\mathrm{Bi}$ ions can be a clue for understanding the properties of the ferroelectricity, piezoelectricity, and/or dielectric properties in Bi-based materials with pseudocubic symmetry. Further studies are needed to reveal that the Bi displacement and the local distortion are directly related to the large electric responses.

Another probable explanation for the large responses in cubic systems could be an electric-field-induced phase transition ${ }^{24-26}$. In such a scenario, the cubic symmetry can theoretically change to a lower symmetry, as exhibited by the splitting of specific XRD peaks. Synchrotron radiation powder XRD (SXRD) experiments have confirmed that an electric-field-induced phase transition does not occur in $0.3 \mathrm{BT}-0.1 \mathrm{BMT}-0.6 \mathrm{BF}$ ceramics ${ }^{27}$. Nevertheless, $0.3 \mathrm{BT}-0.1 \mathrm{BMT}-0.6 \mathrm{BF}$ ceramics with a pseudocubic symmetry distinctly exhibits the most substantial piezoelectric and ferroelectric responses at room temperature among all BT-BMT-BF systems.

Piezoelectric ceramics can deform their dimensions under an applied electric field. In typical ferroelectric ceramics with large piezoelectric responses, the extrinsic contributions of the ferroelectric domain structures play important roles, whereas the intrinsic contributions from the crystal lattice do not have a substantial effect on these responses. In the case of $0.3 \mathrm{BT}-0.1 \mathrm{BMT}-0.6 \mathrm{BF}$ ceramics, all the single-diffraction peaks shift significantly under an applied electric field without peak splitting, which suggests that the dimensions of the crystal lattice can easily change, irrespective of the crystallographic direction in which the electric field is applied. The intrinsic contributions originating purely from the crystal lattice seem to play important roles in the exhibition of the large piezoelectric response in $0.3 \mathrm{BT}-0.1 \mathrm{BMT}-0.6 \mathrm{BF}$ ceramics; this is because particular domain structures cannot be expected owing to their pseudocubic symmetry ${ }^{27}$. In contrast, in both the rhombohedral and tetragonal structures of PZT-based ceramics, the positions of the diffraction peaks related to the polarization directions, i.e., (111) and (002) diffraction peaks, respectively, are almost unchanged under an applied electric field 28,29 . However, the peak positions unrelated to the polarization directions, such as (200) and (111) diffraction peaks, shift significantly. Currently, the so-called intergranular interaction caused by the intrinsic and extrinsic interactions at the ceramics grain boundaries is being proposed to explain the large piezoelectric responses of PZT-based ceramics ${ }^{30}$. Unlike PZT-based ceramics, $0.3 \mathrm{BT}-0.1 \mathrm{BMT}-0.6 \mathrm{BF}$ ceramics is a good target in considering new mechanisms on the emergence of large piezoelectricity.

XRD experiments under external electric fields are effective for revealing the relationships between piezoelectric properties and crystal structures ${ }^{31-36}$. In particular, they are good for evaluating the intrinsic contributions from a crystal lattice.

In this study, the crystal structure of $0.3 \mathrm{BT}-0.1 \mathrm{BMT}-0.6 \mathrm{BF}$ ceramics was investigated at various temperatures by analyzing the SXRD data using the maximum entropy method (MEM)/ Rietveld method ${ }^{37}$ to clarify the emergent mechanisms of piezoelectricity. The visualized three-dimensional electron-density distributions around the Bi ions were not smooth spherical, instead, they were convex-shaped, bulging out in the cubic $\langle 100\rangle$ directions, in the entire temperature range. Therefore, it can be inferred that the Bi ions are off-centered from the corner site of the unit cell with appropriate probabilities. The diffraction patterns obtained under the applied electric field suggested that the large piezoelectric response was a result of the combinational partial ordering of the off-centered $\mathrm{Bi}$ ions toward the electric field. Here, we demonstrated a mechanism for materials with a pseudocubic structure exhibiting large piezoelectric responses.

\section{Results}

SXRD patterns under the applied electric field. Figure 1 shows the SXRD patterns of the synthesized 0.3BT-0.1BMT-0.6BF ceramics with a pseudocubic symmetry. The red line represents the powder diffraction pattern under an applied electric field of $50 \mathrm{kV} \mathrm{cm}^{-1}$, whereas the black line represents the one without an applied electric field. All the diffraction peaks shift toward lower $2 \theta$ angles without any clear peak splitting under the applied electric field, which suggests that the lattice simply expands, maintaining the pseudocubic symmetry. No electric-field-induced phase transition is observed up to $50 \mathrm{kV} \mathrm{cm}^{-1}$. The inset of Fig. 1 displays the $S-E$ curve evaluated by calculating the lattice strain from each diffraction peak from (100) up to (123) peaks and averaging them, which appears to have a typical butterfly shape frequently observed for ferroelectric materials. The $S-E$ curve of Fig. 1 is quite similar to that of $0.3 \mathrm{BT}-0.1 \mathrm{BMT}-0.6 \mathrm{BF}$ ceramics obtained by electrical measurement ${ }^{27}$, which suggests that the main contribution to the large piezoelectric response is from the expansion and contraction of the unit cell, that is, intrinsic contributions. The strains approximated from the inset of Fig. 1 


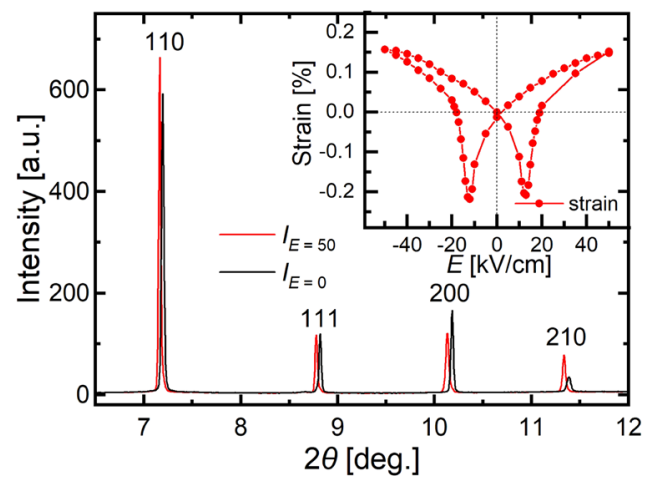

Fig. 1 Synchrotron radiation powder $X$-ray diffraction patterns of the $0.3 \mathrm{BaTiO}_{3}-0.1 \mathrm{Bi}\left(\mathrm{Mg}_{1 / 2} \mathrm{Ti}_{1 / 2}\right) \mathrm{O}_{3}-0.6 \mathrm{BiFeO}_{3}$ ceramic with a pseudocubic symmetry without (black line) and with (red line) an applied electric field of $50 \mathrm{kV} \mathrm{cm}^{-1}$ at room temperature. The lattice strain evaluated from the peak's position is shown in the inset as a function of the electric field.

are as follows: $0.16 \%$ in expansion and $0.24 \%$ in contraction. These values are reasonably large and correspond to approximately two-thirds of those of PZT ceramics under the application of the same electric field ${ }^{38}$. Hence, we can consider that the $0.3 \mathrm{BT}-0.1 \mathrm{BMT}-0.6 \mathrm{BF}$ ceramics has a polar symmetry with an extremely small spontaneous lattice distortion, which can be ignored at room temperature. At this stage, it is unclear why such a ferroelectric material with a negligibly small spontaneous lattice distortion exhibits a large piezoelectric response.

Temperature dependence of the crystal structure. The SXRD patterns of the 0.3BT-0.1BMT-0.6BF ceramics measured at various temperatures are shown in Fig. 2a. The (220) diffraction peak profile is broadened at $700 \mathrm{~K}$ as the temperature is decreased from $1000 \mathrm{~K}$. It appears that a structural phase transition occurs at $T_{\mathrm{C}} \sim 725 \mathrm{~K}$ between 750 and $700 \mathrm{~K}$. A clear peak splitting is observed below $650 \mathrm{~K}$. The peak splitting is the largest at $600 \mathrm{~K}$ below $T_{\mathrm{C}}$. With a further decrease in the temperature, the peak splitting becomes gradually unclear, and the profiles resemble a single peak at low temperatures.

The crystal structure analysis reveals that the high-temperature phase above $T_{\mathrm{C}}$ is cubic with the space group of $P m \overline{3} \mathrm{~m}$ as a prototype structure. It is reported that Bi-based materials, such as BF- and BNT-based materials, form a superlattice by in-phase or antiphase tilting of oxygen octahedra with the space group of $R 3 c$ or $P 4 \mathrm{bm}$ in the ferroelectric phase ${ }^{21,39}$. However, in the case of $0.3 \mathrm{BT}-0.1 \mathrm{BMT}-0.6 \mathrm{BF}$, the superlattice reflections in the ferroelectric phase below $T_{\mathrm{C}}$ cannot be observed, which suggests the validity of a rhombohedral structure with the space group of $R 3 \mathrm{~m}$. The temperature dependence of the lattice parameters assuming a rhombohedral unit cell is presented in Fig. $2 b$ for the entire temperature range of $100-1000 \mathrm{~K}$. The length of the unit cell, $a$, is gradually decreased with decreasing temperature. The phase transition is not noticeable from the changes in the parameter, $a$, while the angle of the unit cell, $\alpha$, significantly deviates from $90^{\circ}$ below $T_{\mathrm{C}}$, clearly indicating a phase transition. The rhombohedral lattice distortion is the largest at $600 \mathrm{~K}$, which is consistent with the largest peak splitting observed in Fig. 2a at the same temperature. The lattice distortion becomes large below $T_{\mathrm{C}}$, and subsequently, with the decrease in temperature, lattice relaxation occurs, which reduces the lattice distortion. The angle, $\alpha$, at $300 \mathrm{~K}$ is $89.97598(7)^{\circ}$, which is very close to $90^{\circ}$. Hence, thus far, we have deduced that the symmetry of $0.3 \mathrm{BT}-0.1 \mathrm{BMT}-0.6 \mathrm{BF}$ at room temperature $(300 \mathrm{~K})$ is cubic.
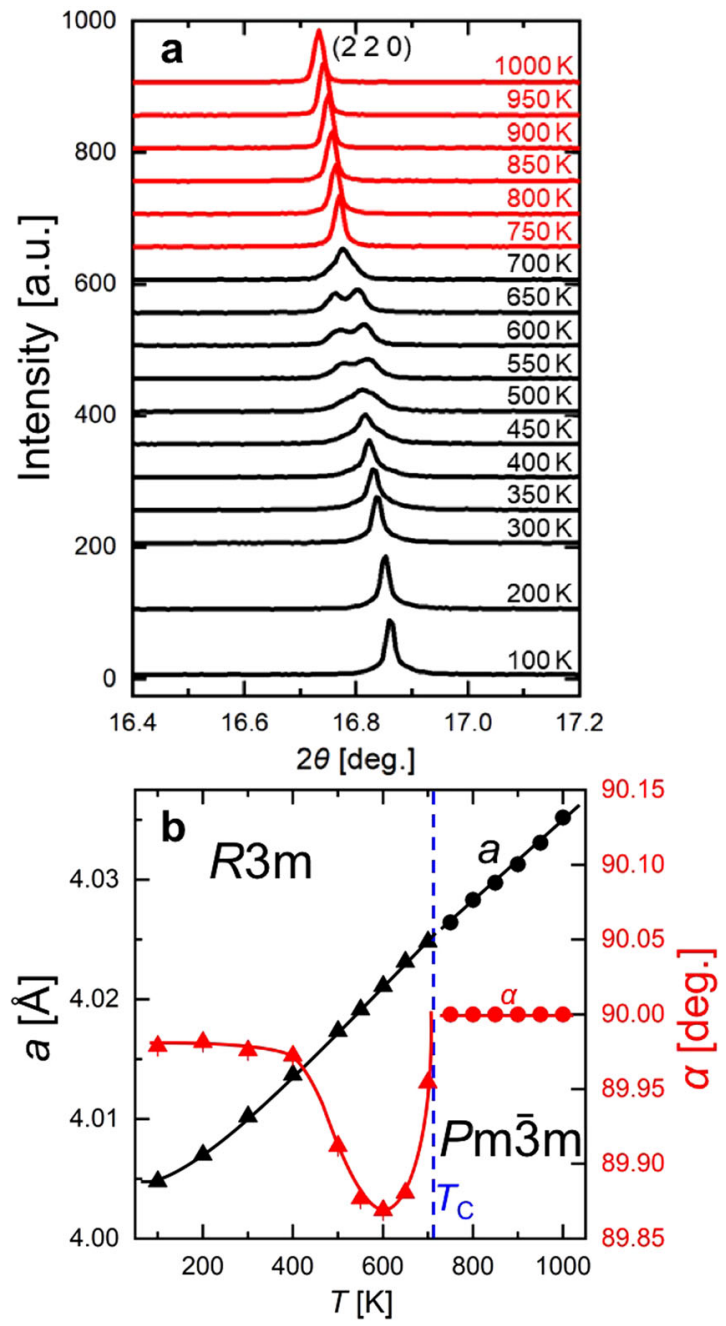

Fig. 2 Temperature dependence of the crystal structure of the $\mathbf{0 . 3 B a T i O}-\mathbf{0 . 1 B i}\left(\mathbf{M g}_{\mathbf{1} / \mathbf{2}} \mathrm{Ti}_{\mathbf{1} / \mathbf{2}}\right) \mathbf{O}_{\mathbf{3}}-\mathbf{- 0 . 6} \mathbf{B i F e O}$ ceramic. a (220) peak profile of the synchrotron radiation powder $\mathbf{X}$-ray diffraction pattern, $\mathbf{b}$ lattice parameters $a$ and $\alpha$ as functions of temperature, assuming a rhombohedral unit cell.

The crystal structure of $0.3 \mathrm{BT}-0.1 \mathrm{BMT}-0.6 \mathrm{BF}$ is analyzed by the MEM/Rietveld method, examining the consistency between the assumed crystal structure model and the electron-density distribution. It is found to have a perovskite-type structure with a general chemical formula, $A B \mathrm{O}_{3}$, where the $A$ sites are occupied by $\mathrm{Ba}$ and $\mathrm{Bi}$ ions and the $B$ sites by $\mathrm{Ti}, \mathrm{Mg}$, and $\mathrm{Fe}$ ions, in the temperature range of 100-1000 K. The crystallographic data with the refined parameters by the Rietveld method at 900,600 , and $300 \mathrm{~K}$ are summarized in Supplementary Tables 1-3, respectively.

The noteworthy structural features revealed are that, in the unit cell, the Bi ion at the $A$ site relaxes to become off-centered in the cubic $\langle 100\rangle$ directions from the special Wyckoff position, i.e., the corner site of $(0,0,0)$ in the unit cell, whereas the $\mathrm{Ba}$ ion at the $A$ site remains at the corner. There is no supporting evidence from the crystallographic data and the structural parameters to suggest that the $\mathrm{Ti}, \mathrm{Mg}$, and $\mathrm{Fe}$ ions at the $B$ sites separately shift to different positions. The Bi ions in the cubic phase, at $900 \mathrm{~K}$, are off-centered by $\sim 0.4 \AA$ at the six equivalent positions in the cubic $\langle 100\rangle$ directions with equal occupancy. The occupancy is changed in the rhombohedral phase. The difference in the rhombohedral lattice distortion originates from the difference in the occupancy. The details are notable in the electron-density distributions, which are described in the next section. 


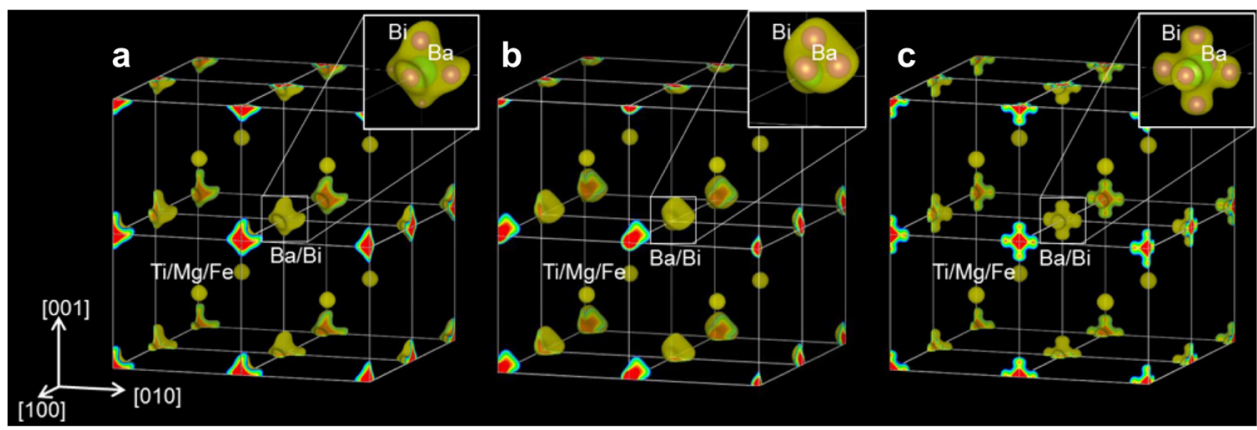

Fig. 3 Electron-density distributions of $0.3 \mathrm{BaTiO}_{3}-0.1 \mathrm{Bi}\left(\mathrm{Mg}_{1 / 2} \mathrm{Ti}_{1 / 2}\right) \mathrm{O}_{3}-0.6 \mathrm{BiFeO}$ visualized by the maximum entropy method at an isosurface value of $\mathbf{5 0}$ e $\AA^{-\mathbf{3}}$ for $\mathbf{2} \times \mathbf{2} \times \mathbf{2}$ unit cells. The electron-density distribution at a $300 \mathrm{~K}$ (ferroelectric phase), b $600 \mathrm{~K}$ (ferroelectric phase), and c $900 \mathrm{~K}$ (paraelectric phase).
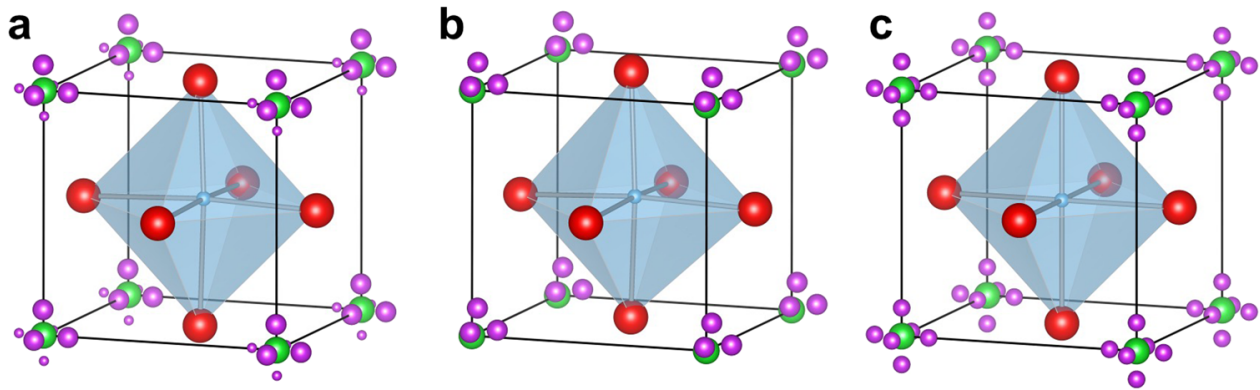

Fig. 4 Schematics of the crystal structure of $0.3 \mathrm{BaTiO}_{\mathbf{3}}-\mathbf{0 . 1 B i}\left(\mathrm{Mg}_{\mathbf{1 / 2}} \mathbf{T i}_{\mathbf{1} / \mathbf{2}}\right) \mathrm{O}_{\mathbf{3}}-\mathbf{- 0 . 6 \mathrm { BiFeO }}$ as a function of temperature. The crystal structures at a $300 \mathrm{~K}$ (the off-centered $\mathrm{Bi}$ ions are partially ordered at six sites with a polar symmetry), $\mathbf{b} 600 \mathrm{~K}$ (the off-centered Bi ions are partially ordered at three sites with a polar symmetry), and c $900 \mathrm{~K}$ (the off-centered Bi ions are randomly disordered at six sites with a nonpolar symmetry).

Visualization of off-centered $\mathbf{B i}$ ions. Figure 3 presents the electron-density distributions of $0.3 \mathrm{BT}-0.1 \mathrm{BMT}-0.6 \mathrm{BF}$ in the ferroelectric phase (rhombohedral at 300 and $600 \mathrm{~K}$ ) and the paraelectric phase (cubic at $900 \mathrm{~K}$ ), which were calculated by MEM using the program ENIGMA ${ }^{40}$. The MEM analysis was performed with the unit cell divided into small voxels. The volume of one voxel corresponded to approximately $0.05 \times 0.05 \times$ $0.05 \AA^{3}$. The figures are drawn at a comparatively high isosurface level of $50 \mathrm{e} \AA^{-3}$, to emphasize the shapes of the inner electron clouds with high density around the $A$ - and $B$-sites. Consequently, the electron-density distributions around the light ions, i.e., $\mathrm{O}$ ions, are not observed in the figures.

As seen in Fig. 3, the electron-density distributions around the $B$ sites $(\mathrm{Ti} / \mathrm{Mg} / \mathrm{Fe})$ are spherically isotropic. It can be considered that the $\mathrm{Ti}, \mathrm{Mg}$, and $\mathrm{Fe}$ ions remain at the same positions at the $B$ sites. However, the electron-density distributions around the $A$ sites $(\mathrm{Ba} / \mathrm{Bi})$ are extremely anisotropic in the $\langle 100\rangle$ directions, and it is reasonably unexpected for the $\mathrm{Ba}$ and $\mathrm{Bi}$ ions to remain located at the same positions. Finally, a very close agreement between the structure model refined by the Rietveld method and the MEM electron-density distribution can be achieved. In the model, only the $\mathrm{Bi}$ ion is off-centered from the corner site in the unit cell in the $\langle 100\rangle$ directions, whereas the $\mathrm{Ba}$ ion remains at the corner. In the paraelectric phase at $900 \mathrm{~K}$, as shown by the enlarged electron- density distribution at the $A$ site in Fig. $3 c$, the $\mathrm{Bi}$ ions are disordered being off-centered at the six sites in the $\langle 100\rangle$ directions. In the ferroelectric phase at $600 \mathrm{~K}$, the $\mathrm{Bi}$ ions are partially ordered at the three sites in the [100], [010], and [001] directions with the same occupancy, as displayed in Fig. 3b. The rhombohedral lattice distortion is caused by the partial ordering of the Bi ions at the three sites. Therefore, spontaneous polarization emerges toward the cubic [111] direction. However, in the ferroelectric phase at $300 \mathrm{~K}$, as presented in Fig. 3a, the Bi ions occupy the three sites in the $[\overline{1} 00],[0 \overline{1} 0]$, and $[00 \overline{1}]$ directions in addition to the three sites in the [100], [010], and [001] directions. The occupancies of the $\mathrm{Bi}$ ions at the former three sites are smaller than those at the latter three sites, which suppresses the rhombohedral lattice distortion. The former and latter Bi ions are labeled in Supplementary Table 3 as Bi2 and Bi1 ions, respectively. The pseudocubic symmetry observed in the $0.3 \mathrm{BT}-0.1 \mathrm{BMT}-0.6 \mathrm{BF}$ ceramics at room temperature $(300 \mathrm{~K})$ originated from the structural characteristics of the $\mathrm{Bi}$ ions occupying the six sites as well as those in the paraelectric phase.

Combinational partial ordering of ions associated with phase transitions is proposed to occur in pure BT and BT-based ferroelectrics with perovskite-type structures. The dominant view is that in the cubic paraelectric phase, the Ti ions at the $B$ sites are off-centered at the eight sites in the $\langle 111\rangle$ directions ${ }^{41-44}$. In the tetragonal ferroelectric phase, the $\mathrm{Ti}$ ions are suggested to be partially ordered at the four sites in the [111], [111], [111], and [111] directions with the same occupancy, associated with a phase transition $^{42,45}$. Hence, in the tetragonal structure, spontaneous polarization emerges toward the [001] direction. Unlike in the pure BT and BT-based ferroelectrics, the occupancy in the partial ordering in $0.3 \mathrm{BT}-0.1 \mathrm{BMT}-0.6 \mathrm{BF}$ can be changed with temperature, in a particular ferroelectric phase. Recently, in solid solutions including $\mathrm{Bi}$ ions, such as $\mathrm{BF}-\mathrm{BT}^{46,47}$ and $\mathrm{BNT}^{48}$, offcentering of the Bi ions was observed. It was reported that the change in the Bi sites from six to ten split sites involved a phase transition from paraelectric (cubic) to ferroelectric phases (tetragonal) in $\mathrm{K}_{0.5} \mathrm{Bi}_{0.5} \mathrm{TiO}_{3}{ }^{22}$. However, the partial ordering under an applied electric field is not discussed in the ferroelectric phase. We will discuss the partial ordering of $\mathrm{Bi}$ ions under the electric field in $0.3 \mathrm{BT}-0.1 \mathrm{BMT}-0.6 \mathrm{BF}$ in the "Discussion".

The structural characteristics of $0.3 \mathrm{BT}-0.1 \mathrm{BMT}-0.6 \mathrm{BF}$ revealed in this study are schematically summarized in Fig. 4 by VESTA ${ }^{49}$ 

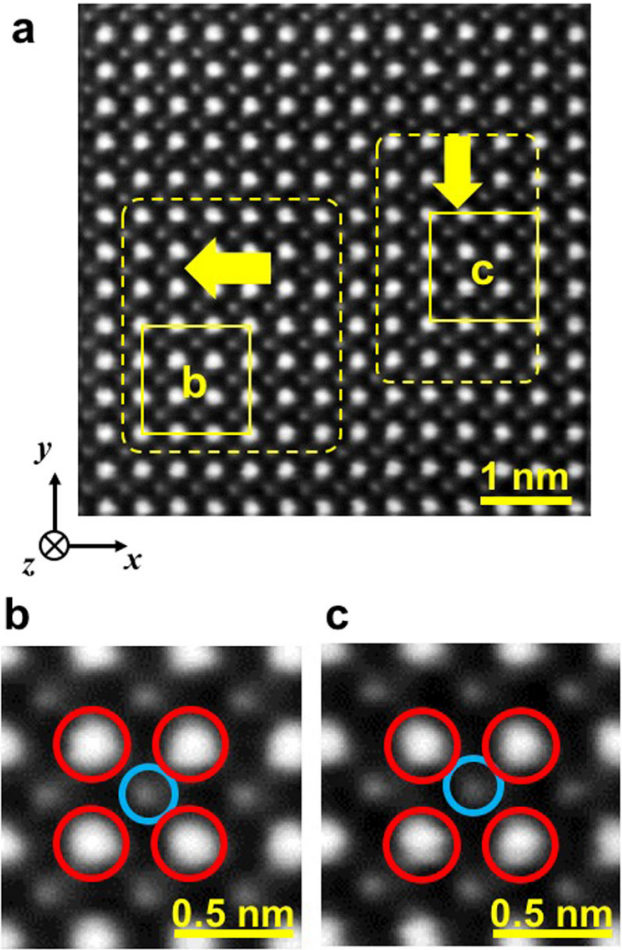

Fig. 5 STEM image of the $0.3 \mathrm{BaTiO}_{3}-0.1 \mathrm{Bi}\left(\mathrm{Mg}_{1 / 2} \mathrm{Ti}_{1 / 2}\right) \mathrm{O}_{3}-0.6 \mathrm{BiFeO}$ ceramics. a Overall image, $\mathbf{b}$ and $\mathbf{c}$ enlarged images of areas $\mathbf{b}$ and $\mathbf{c}$ in $\mathbf{a}$, respectively, indicating the $\mathrm{Bi}$ ions by red circles and the $\mathrm{B}$-site ion by a blue circle. In the areas surrounded by yellow dots, including areas $\mathbf{b}$ and $\mathbf{c}$ in $\mathbf{a}$, the $\mathrm{Bi}$ ions shift to left and lower sides, respectively, relative to the Bsite ions.

with a ball-and-stick model, which should be discussed in comparison with the three-dimensional electron-density distributions presented in Fig. 3. In the case of $0.3 \mathrm{BT}-0.1 \mathrm{BMT}-0.6 \mathrm{BF}$, it is unique that in the ferroelectric phase, the degree of partial ordering at the off-centered Bi-ion sites, as shown in Fig. $4 \mathrm{a}$, b, can be easily controlled by external stimuli, such as changes in the temperatures, and may even be the electric field.

The off-centering of $\mathrm{Bi}$ ions was detected via scanning transmission electron microscopy (STEM). Figure 5a presents the STEM image of $0.3 \mathrm{BT}-0.1 \mathrm{BMT}-0.6 \mathrm{BF}$ at room temperature $(300 \mathrm{~K})$, wherein the $\mathrm{Bi}$ ions located at the $A$ sites form a cluster in the region of several $\mathrm{nm}^{2}$. In the areas, including areas (b) and (c), all the $A$-site ions shift simultaneously left and to lower sides, respectively, relative to the $B$-site ions (Supplementary Fig. 1). The average magnitude of the displacement and the directions of the $A$ site ions are $\sim 0.14 \AA$ and $\langle 100\rangle$ directions, respectively, in the cluster region. The smaller magnitude than that obtained by the Rietveld refinement is owing to the possible overlapping of the clusters of $\mathrm{Bi}$ ions with different off-centered directions along the incident direction of the electron beam and the mixing of the oncentered $\mathrm{Ba}$ and off-centered $\mathrm{Bi}$ ions. The STEM image suggests that the $\mathrm{Bi}$ ions are not dynamically disordered in the $\langle 100\rangle$ directions, but are statically ordered at an appropriate position in one of the $\langle 100\rangle$ directions.

\section{Discussion}

We have demonstrated that the $\mathrm{Bi}$ ions in the 0.3BT-0.1BMT$0.6 \mathrm{BF}$ ceramics prefer to relax to off-centered positions in the $\langle 100\rangle$ directions from the corner of the unit cell, and the occupancies of the $\mathrm{Bi}$ ions at these off-centered positions can be changed by varying the temperature in the ferroelectric rhombohedral phase
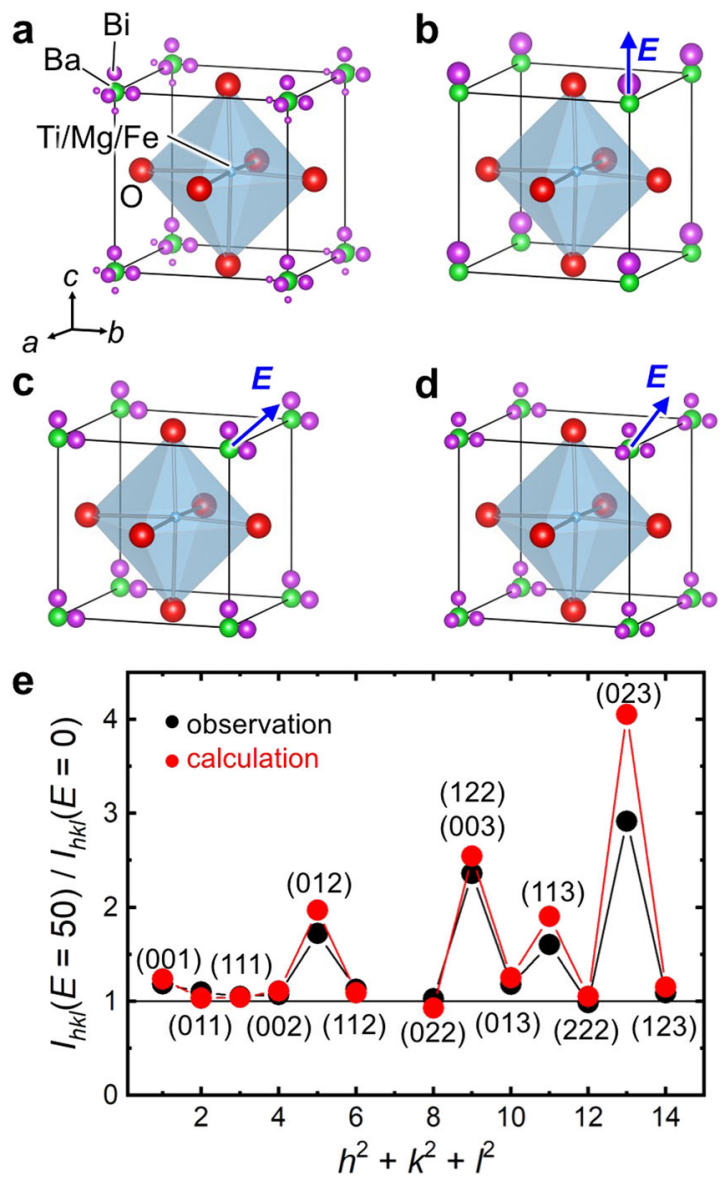

Fig. 6 Schematics of the crystal structures under an applied electric field. a Crystal structure without an electric field (crystal structure refined at $300 \mathrm{~K}$ ). The proposed structural models under an electric field of $50 \mathrm{kV} \mathrm{cm}^{-1}$ for b E // [001], c E // [011], and d E // [111]. e Calculated and observed intensity ratios of the diffraction peaks with $\left(50 \mathrm{kV} \mathrm{cm}^{-1}\right)$ and without an electric field.

while maintaining the crystal symmetry. Based on these structural characteristics, we propose a structural model under an applied electric field to understand the reason the $0.3 \mathrm{BT}-0.1 \mathrm{BMT}-0.6 \mathrm{BF}$ ceramics with a pseudocubic symmetry exhibits piezoelectric and ferroelectric responses.

As presented in Fig. 1, the diffraction peaks shift with their profiles remaining almost unchanged, under the applied electric field. However, it is noteworthy that their intensities are significantly modified. Some peaks become more intense, whereas the others are weakened. The ratio of the change for the (210) peak is the largest, as can be seen from Fig. 1. These results indicate that the structure factors are changed under the applied electric field.

From the experimental findings that the occupancies of the Bi ions at the off-centered positions in the $\langle 100\rangle$ directions are different at 600 and $300 \mathrm{~K}$, as shown in Fig. 4, we propose a simple structural model in which only the occupancies of the $\mathrm{Bi}$ ions vary according to the direction of the applied electric field, as displayed in Fig. 6b-d. In the model, we assume that the Bi ions essentially prefer the off-centered positions in the $\langle 100\rangle$ directions, regardless of the direction of the applied electric field. Furthermore, the magnitudes of the displacements of the offcentered $\mathrm{Bi}$ ions do not change with or without the electric field. As presented in Fig. $6 \mathrm{a}$, at $300 \mathrm{~K}$, under the condition of no applied electric field, the disordered $\mathrm{Bi}$ ions at the three 
off-centered positions in the [100], [010], and [001] directions have higher occupancies, whereas those at the other three positions in the $[\overline{1} 00],[0 \overline{1} 0]$, and $[00 \overline{1}]$ directions have lower occupancies. If the electric field is applied in the [001] direction, as shown in Fig. 6b, our model proposes that the Bi ions are fully ordered at the positions in the [001] direction. In the cases when the electric field is applied in the [011] and [111] directions, the $\mathrm{Bi}$ ions are partially ordered at the two positions in the [010] and [001] directions and at the three positions in the [100], [010], and [001] directions with the same occupancy, as displayed in Fig. 6c, $\mathrm{d}$, respectively. Therefore, the direction of the vector sum of the three vectors in the off-centered [100], [010], and [001] directions weighted by the occupancies of the $\mathrm{Bi}$ ions is in the direction of the electric field.

Based on the structural models introduced in Fig. 6, we calculated the structural factors with and without an electric field for each $(h k l)$ diffraction to evaluate the intensities of the diffraction peaks, which are proportional to the square of the structure factors. The calculated intensity ratio for the structural model, such as Fig. $6 \mathrm{~b}-\mathrm{d}$, under the electric field can be expressed as follows:

$$
\left(\frac{I_{h k l(E=50)}}{I_{h k l(E=0)}}\right)_{c a l .}=\frac{n_{1} I_{h k l}\left(B i_{1-s i t e}\right)+n_{2} I_{h k l}\left(B i_{2-s i t e}\right)+n_{3} I_{h k l}\left(B i_{3-s i t e}\right)}{I_{h k l}^{\prime}},
$$

where $I_{h k l}^{\prime}$ is the intensity for the six-site model (Fig. 6a) without the electric field at $300 \mathrm{~K} . I_{h k l}\left(\mathrm{Bi}_{x-\text { site }}\right)$ indicates the intensity of the $(h k l)$ peak calculated from the structural model in which Bi ions occupy off-centered sites $x(x=1,2$, and 3$)$ in the [100], [010], and [001] directions with appropriate occupancies, respectively, and $n_{x}$ indicates the ratio of intensity contributions $\left(n_{1}+n_{2}+n_{3}=1\right)$ weighted by the occupancies of the Bi ions. The calculated intensity ratios for the structure models are indicated in Fig. 6e by red circles. The observed values indicated by black circles in the figure are evaluated from the experimentally measured intensities for each diffraction by tilting the sample ceramic with respect to the incident X-rays so that each scattering vector is parallel to the direction of the applied electric field of 50 $\mathrm{kV} \mathrm{cm}^{-1}$. The tendency of the index dependence of the observed values, as presented in Fig. 6 e, is very similar to that of the calculated values. Therefore, we consider that the proposed structural model under an applied electric field is appropriate. It is reasonable to consider that the proposed ordering variations of the $\mathrm{Bi}$ ions under an electric field are strongly related to the piezoelectric and ferroelectric responses in the pseudocubic $0.3 \mathrm{BT}-0.1 \mathrm{BMT}-0.6 \mathrm{BF}$ ceramics.

In typical ferroelectrics, the electric-field-induced strain originates from the intrinsic lattice strain of the unit cell and the extrinsic domain reorientation. The applied electric field basically induces expansion and contraction of the unit cell of the crystal lattice, generating intrinsic lattice strain, and therefore, resulting in a shift of the XRD peak positions. Regarding the extrinsic effect, when the electric field is applied to a ceramic with spontaneous polarization parallel to the [001] direction in the tetragonal symmetry, such as BT ceramics, the intensity ratio between the specific $(001)$ and $(100) /(010)$ peaks may be modified by the $90^{\circ}$ domain reorientation. Hence, XRD experiments enable the individual detection of the intrinsic and extrinsic contributions to the piezoelectric response under an applied electric field. However, for the $0.3 \mathrm{BT}-0.1 \mathrm{BMT}-0.6 \mathrm{BF}$ ceramics having a pseudocubic symmetry, such $90^{\circ}$ domain reorientation essentially cannot be detected even via SXRD.

Thus far, $\mathrm{BT}-\mathrm{ABO}_{3}-\mathrm{BF}$ solid solutions have been known to be relaxor ferroelectrics with a pseudocubic symmetry ${ }^{50-53}$, which exhibits slim $P-E$ hysteresis loops. The $S-E$ curves are characterized

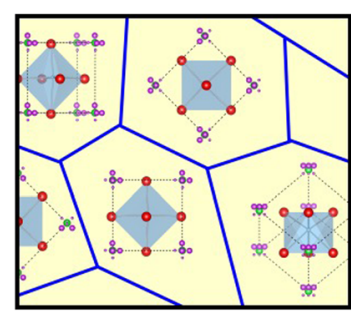

$E=0$

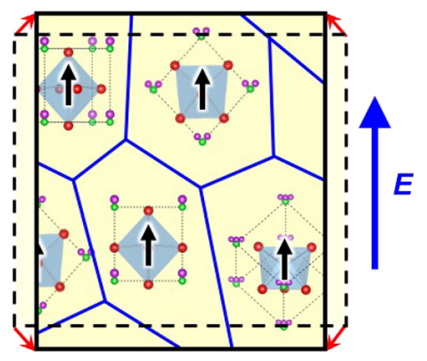

$E \neq 0$
Fig. 7 Micro- and macroimages of the ceramic grains in the $0.3 \mathrm{BaTiO}_{3}-\mathbf{0 . 1 B i}\left(\mathrm{Mg}_{\mathbf{1} / \mathbf{2}} \mathrm{Ti}_{\mathbf{1} / \mathbf{2}}\right) \mathrm{O}_{\mathbf{3}}-\mathbf{0 . 6} \mathrm{BiFeO}_{3}$ ceramic. The ceramic grains without $(E=0)$ and with $(E \neq 0)$ under an electric field.

by indicating zero and/or very small strains on the negative strain side of the curves. It might be to occur the piezoelectric and ferroelectric responses attributed to the reorientation of polar domains with subnanometer sizes ${ }^{54}$. However, 0.3BT-0.1BMT-0.6BF ceramics exhibits typical ferroelectricity with the square-shaped $P-E$ hysteresis loops and the butterfly-shaped $S-E$ curves $^{13,27}$. In general, there are no ferroelectric domains in cubic crystals. The piezoelectric response is very small if there is no contribution from the domains. Therefore, it was not clear why the $0.3 \mathrm{BT}-0.1 \mathrm{BMT}-0.6 \mathrm{BF}$ ceramic exhibited a piezoelectric response as a typical ferroelectric.

Ceramics are formed of numerous small ceramic grains, which is also the case of the $0.3 \mathrm{BT}-0.1 \mathrm{BMT}-0.6 \mathrm{BF}$ ceramics with ceramic grains of $1-2 \mu \mathrm{m} \operatorname{sizes}^{55}$, as illustrated in Fig. 7. If the grain size is extremely small, a grain may consist of a single crystal, and the crystal lattice is well arranged in the grain. Note that the orientation of the crystal lattice in a particular grain is different from those in other grains. In $0.3 \mathrm{BT}-0.1 \mathrm{BMT}-0.6 \mathrm{BF}$ ceramics, the $\mathrm{Bi}$ ions can easily change their off-centered positions in the directions close to the applied electric field. The unit cell in the grain is expanded in the direction of the electric field by the combinational partial ordering of the Bi ions under the electric field. Because all the grains are expanded in the direction of the electric field, as shown in Fig. 7, the ceramic is macroscopically expanded in the direction, resulting in the large piezoelectric response. The characteristic of the $0.3 \mathrm{BT}-0.1 \mathrm{BMT}-0.6 \mathrm{BF}$ ceramic is that all the ceramic grains are active for the response under an electric field. The change in atomic orientation that $\mathrm{Bi}$ ions ordered on a side under the electric field are ordered on the opposite side when the direction of the electric field is reversed can be regarded as a type of domain switching to show polarization reversal in ferroelectricity. Therefore, we consider that typical piezoelectric and ferroelectric responses are observed in the $S-E$ curves and the $P-E$ hysteresis loops of $0.3 \mathrm{BT}-0.1 \mathrm{BMT}-0.6 \mathrm{BF}$ ceramics, respectively. The reason the $\mathrm{Bi}$ ions are off-centered and can easily change their positions is that in addition to the $\mathrm{Bi}^{3+}$ ions possessing lone electron pairs, the $\mathrm{Ba}^{2+}$ ions, having ionic radii $(1.61 \AA)$ larger than those of $\mathrm{Bi}^{3+}$ ions $(1.45 \AA)$, broaden the space around the $A$ sites on an average, allowing easy displacement of the Bi ions.

Numerous materials are referred to as pseudocubic. In ferroelectrics, when a ferroelectric response is observed in those with cubic symmetry, we generally use this term to resolve the contradiction between its physical properties and crystal structure. The $\mathrm{Pb}$-free $0.3 \mathrm{BT}-0.1 \mathrm{BMT}-0.6 \mathrm{BF}$ exhibits typical piezoelectric and ferroelectric responses in spite of having a pseudocubic structure. The SXRD experiments with and without the electric field reveal that the significant piezoelectric response is caused by the combinational partial ordering of the off-centered $\mathrm{Bi}$ ions in any $\langle 100\rangle$ directions in which the electric field is applied. Many of the supposed pseudocubic materials may have similar mechanisms for the emergence of their piezoelectric and ferroelectric 
properties. These types of unique ferroelectrics exhibit significant properties only when an electric field is applied. If there is no applied electric field, then the exhibition of a large ferroelectric response cannot be predicted. The calculated spontaneous polarization without an electric field is extremely small to predict the large response under an electric field. We propose that the key concept to develop such ferroelectrics is to use ions that tend to fluctuate and become off-centered, such as a $\mathrm{Bi}^{3+}$ ion with lone electron pairs, and to locate the ions in sufficient space at the $A$ site expanded by other ions. The proposed mechanism can provide information essential for designing $\mathrm{Pb}$-free ceramics that deform well in all directions by controlling the spatial distributions of the fluctuated and off-centered ions under an applied electric field.

\section{Methods}

Materials and characterization. The $0.3 \mathrm{BT}-0.1 \mathrm{BMT}-0.6 \mathrm{BF}$ ceramics was fabricated using a conventional solid-state reaction method. The raw powders used were $\mathrm{Bi}_{2} \mathrm{O}_{3}$ (particle size: $5 \mu \mathrm{m}$, Rare Metallic), $\mathrm{MgO}$ (particle size: $5 \mu \mathrm{m}$, Rare Metallic), $\mathrm{TiO}_{2}$ (particle size: $7 \mathrm{~nm}$, Ishihara Sangyo), $\mathrm{BaTiO}_{3}$ (BT01, particle size: $0.1 \mu \mathrm{m}$, Sakai Chemical Co. Ltd.), and $\mathrm{BiFeO}_{3}$ (particle size: $0.49 \mu \mathrm{m}$, Nippon Chemical Industrial Co. Ltd.). The raw powders were mixed with zirconia balls and ethanol, and subsequently ball-milled for $16 \mathrm{~h}$ at a ball-milling speed of $250 \mathrm{rpm}$. The resultant ball-milled slurry was dried in a drying oven and calcined at $1173 \mathrm{~K}$ for 6 h. Approximately $0.05 \mathrm{wt} \% \mathrm{MnO}_{2}$ was added to the calcined powders, and ball milling was performed again followed by drying. Finally, polyvinyl butyral (PVB) was added as a binder to the resultant powder, and disk-type green pellets were formed by uniaxial pressing. The green pellets were then sintered at $1273 \mathrm{~K}$ for $2 \mathrm{~h}$ after binder removal treatment at $973 \mathrm{~K}$ for $10 \mathrm{~h}$.

Synchrotron radiation X-ray diffraction. High-energy SXRD experiments were conducted in the BL02B2 beamline in SPring-8 under various temperatures and electric fields. For the measurement of the temperature dependence of the crystal structure, the sintered ceramic was used in powder form, which was sealed in a quartz capillary with $0.1-\mathrm{mm}$ diameter. The lattice parameters were analyzed by the Rietveld method in the temperature range of $100-1000 \mathrm{~K}$ using the SXRD patterns measured by a MYTHEN (Dectris) detector. The energy of the X-rays used was 30 $\mathrm{keV}(0.41236(1) \AA)$. The electron-density distributions were visualized by the MEM/Rietveld method ${ }^{37}$ at 300,600 , and $900 \mathrm{~K}$ for the SXRD patterns recorded on an Imaging Plate (Fuji Film). The energy of the X-rays used was $35 \mathrm{keV}(0.35433$ (1) $\AA$ ).

For the measurements under an applied electric field, a ceramic bar with dimensions of $0.2 \mathrm{~mm}$ (thickness) $\times 0.3 \mathrm{~mm}$ (width) $\times 8 \mathrm{~mm}$ (length) was used. The bar-type sample was annealed at $1073 \mathrm{~K}$ for $20 \mathrm{~h}$ and quenched in water. Gold electrodes were coated on both polished sides $(0.3 \times 8 \mathrm{~mm})$ and then annealed at $537 \mathrm{~K}$ for $10 \mathrm{~min}$. The SXRD patterns were measured using X-rays of $35 \mathrm{keV}$ (0.35433(1) $\AA$ ) by the MYTHEN detector. The sample was set in the sample holder such that the longitudinal direction was perpendicular to the incident X-rays. Each intensity of the $(h k l)$ diffraction was measured by tilting the sample at the appropriate angle with respect to the incident X-rays so that the scattering vector of each diffraction was parallel to the direction of the applied electric field, e.g., $\omega=$ $10.16^{\circ}$ for $(002)$. The sample was rocked around the longitudinal direction at $\pm 2.5^{\circ}$ during the measurements. Under these experimental conditions, we succeeded in measuring data up to $2 \theta=20^{\circ}$ in the direction of the electric field, where the (123) peak was observed. For the simultaneous measurement of all the diffraction patterns, the tilting angle was fixed as the angle for (002). The electric field in the range of -50 to $+50 \mathrm{kV} \mathrm{cm}^{-1}$ was applied with bipolar cycling using a PSX-12B (Matsushige Precision Co., Ltd.) current power supply, and the voltage was amplified by 1000 times using an HVA 4321 (NF Corporation) amplifier.

Scanning transmission electron microscopy. STEM was conducted using a JEMARM200F ACCELARM microscope (JEOL Ltd.) operated at an acceleration voltage of $200 \mathrm{kV}$. Annular dark-field (ADF) images were recorded using DigiscanII (Gatan Inc.) with a dwell time of $\sim 31.5 \mu$ s and a flyback of $500 \mu$ s. A probe-forming aperture semiangle of $\sim 26 \mathrm{mrad}$ and a detection angular range of $68-270 \mathrm{mrad}$ were used for the imaging. The image distortion caused by the combination of the sample drift and the scanning system in the microscope ${ }^{56}$ was corrected using the JitterBug plug-in ${ }^{57}$ (HREM Research Inc.) in the DigitalMicrograph software. Accordingly, the average lattice parameters of the unit cells observed in the images became consistent with those measured via SXRD. The obtained images were smoothened via radial Wiener filtering (HREM Research Inc.). The positions of the $A$ - and $B$-site cations in the images were determined using the two-dimensional Gaussian fitting method ${ }^{58}$ to calculate the displacements of the $A$-site cations relative to the centers of the unit cells formed of $B$-site cations.

\section{Data availability}

The authors declare that obtained and analyzed data are contained in the paper and the Supplementary Information, and are available from the corresponding author on reasonable request.

Received: 3 March 2020; Accepted: 11 September 2020;

Published online: 06 October 2020

\section{References}

1. Jaffe, B., Cook, W. R., \& Jaffe, H. Piezoelectric Ceramics (Aocademic, 1971).

2. Damjanovic, D. A morphotropic phase boundary system based on polarization rotation and polarization extension. Appl. Phys. Lett. 97, 062906 (2010).

3. Fu, H. \& Cohen, R. E. Polarization rotation mechanism for ultrahigh electromechanical response in single-crystal piezoelectrics. Nature $\mathbf{4 0 3}$, 281-283 (2000).

4. Ahart, M. et al. Origin of morphotropic phase boundaries in ferroelectrics. Nature 451, 545-548 (2008).

5. Shrout, T. R. \& Zhang, S. J. Lead-free piezoelectric ceramics: alternatives for PZT? J. Electroceram. 19, 111-124 (2007).

6. Rödel, J. et al. Transferring lead-free piezoelectric ceramics into application. $J$ Euro. Ceram. Soc. 35, 1659-1681 (2015)

7. Rödel, J., Jo, W., Seifert, K. T. P., Anton, E.-M. \& Granzow, T. Perspective on the development of lead-free piezoceramics. J. Am. Ceram. Soc. 92, 1153-1177 (2009)

8. Lee, M. H. et al. High-performance lead-free piezoceramics with high Curie temperatures. Adv. Mater. 27, 6976-6982 (2015).

9. Dong, G., Fan, H., Shi, J. \& Li, M. Composition- and temperature-dependent large strain in $(1-x)\left(0.8 \mathrm{Bi}_{0.5} \mathrm{Na}_{0.5} \mathrm{TiO}_{3}-0.2 \mathrm{Bi}_{0.5} \mathrm{~K}_{0.5} \mathrm{TiO}_{3}\right)-x \mathrm{NaNbO}_{3}$ ceramics. J. Am. Ceram. Soc. 98, 1150-1155 (2015).

10. Kim, S. et al. A correlation between piezoelectric response and crystallographic structural parameter observed in lead-free $(1-x)\left(\mathrm{Bi}_{0.5} \mathrm{Na}_{0.5}\right) \mathrm{TiO}_{3}-x \mathrm{SrTiO}_{3}$ piezoelectrics. J. Euro. Ceram. Soc. 37, 1379-1386 (2017).

11. Singh, A. \& Chatterjee, R. Structural, electrical, and strain properties of stoichiometric 1- $x-y\left(\mathrm{Bi}_{0.5} \mathrm{Na}_{0.5}\right) \mathrm{TiO}_{3}-x\left(\mathrm{Bi}_{0.5} \mathrm{~K}_{0.5} \mathrm{TiO}_{3}\right)-\mathrm{y}\left(\mathrm{Na}_{0.5} \mathrm{~K}_{0.5}\right) \mathrm{NbO}_{3}$ solid solutions. J. Appl. Phys. 109, 024105 (2011).

12. Fujii, I. et al. Effect of sintering condition and V-doping on the piezoelectric properties of $\mathrm{BaTiO}_{3}-\mathrm{Bi}\left(\mathrm{Mg}_{1 / 2} \mathrm{Ti}_{1 / 2}\right) \mathrm{O}_{3}-\mathrm{BiFeO}_{3}$ ceramics. J. Ceram. Soc. Jpn. 121, 589-592 (2013).

13. Nam, $\mathrm{H}$. et al. Influence of quenching temperature on piezoelectric and ferroelectrics properties in $\mathrm{BaTiO}_{3}-\mathrm{Bi}\left(\mathrm{Mg}_{1 / 2} \mathrm{Ti}_{1 / 2}\right) \mathrm{O}_{3}-\mathrm{BiFeO}_{3}$ ceramics. Ceramics Int. 44, S199-S202 (2018).

14. Fujii, I. et al. Structural, dielectric and piezoelectric properties of Mn-doped $\mathrm{BaTiO}_{3}-\mathrm{Bi}\left(\mathrm{Mg}_{1 / 2} \mathrm{Ti}_{1 / 2}\right) \mathrm{O}_{3}-\mathrm{BiFeO}_{3}$ ceramics. Jpn. J. Appl. Phys. 50, 09ND07 (2011).

15. Zhang, S. T., Kounga, A. B., Aulbach, E., Ehrenberg, H. \& Rödel, J. Giant strain in lead-free piezoelectrics $\mathrm{Bi}_{0.5} \mathrm{Na}_{0.5} \mathrm{TiO}_{3}-\mathrm{BaTiO}_{3}-\mathrm{K}_{0.5} \mathrm{Na}_{0.5} \mathrm{NbO}_{3}$ system. Appl. Phys. Lett. 91, 112906 (2007).

16. Kim, S. et al. Structural and electrical characteristics of potential candidate lead-free $\mathrm{BiFeO}_{3}-\mathrm{BaTiO}_{3}$ piezoelectric ceramics. J. Appl. Phys. 122, 164105 (2017).

17. Obilor, U., Pascual-Gonzalez, C., Murakami, S., Reaney, I. M. \& Feteira, A. Study of the temperature dependence of the giant electric field-induced strain in Nb-doped BNT-BT-BKT piezoceramics. Mater. Res. Bull. 97, 385-392 (2018).

18. Krayzman, V., Levin, I., Woicik, J. C. \& Bridges, F. Correlated rattling-ion origins of dielectric properties in reentrant dipole glasses $\mathrm{BaTiO}_{3}-\mathrm{BiScO}_{3}$. Appl. Phys. Lett. 107, 192903 (2015)

19. Usher, T.-M., Levin, I., Daniels, J. E. \& Jones, J. L. Electric-field-induced local and mesoscale structural changes in polycrystalline dielectrics and ferroelectrics. Sci. Rep. 5, 14678 (2015).

20. Keeble, D. S. et al. Bifurcated polarization rotation in bismuth-based piezoelectrics. Adv. Funct. Mater. 23, 185-190 (2013).

21. Luo, C. et al. Neutron and $x$-ray scattering study of phonon dispersion and diffuse scattering in $(\mathrm{Na}, \mathrm{Bi}) \mathrm{TiO}_{3}-x \mathrm{BaTiO}_{3}$ single crystals near the morphotropic phase boundary. Phys. Rev. B 96, 174108 (2017).

22. Levin, I. et al. Nanoscale polar heterogeneities and branching Bi-displacement directions in $\mathrm{K}_{0.5} \mathrm{Bi}_{0.5} \mathrm{TiO}_{3}$. Chem. Mater. 31, 2450-2458 (2019).

23. Wang, G. et al. Origin of the large electrostrain in $\mathrm{BiFeO}_{3}-\mathrm{BaTiO}_{3}$ based leadfree ceramics. J. mater. Chem. A 7, 21254-21263 (2019).

24. Chen, C. et al. Giant strain and electric-field-induced phase transition in leadfree $\left(\mathrm{Na}_{0.5} \mathrm{Bi}_{0.5}\right) \mathrm{TiO}_{3}-\mathrm{BaTiO}_{3}-\left(\mathrm{K}_{0.5} \mathrm{Na}_{0.5}\right) \mathrm{NbO}_{3}$ single crystal. Appl. Phys. Lett. 108, $022903(2016)$

25. Daniels, J. E., Jo, W., Rödel, J. \& Jones, J. L. Electric-field-induced phase transformation at a lead-free morphotropic phase boundary: Case study in a 
93\% $\left(\mathrm{Bi}_{0.5} \mathrm{Na}_{0.5}\right) \mathrm{TiO}_{3}-7 \% \mathrm{BaTiO}_{3}$ piezoelectric ceramic. Appl. Phys. Lett. 95, 032904 (2009).

26. Luo, C. et al. Crystallographic direction dependence of direct current field induced strain and phase transitions in $\mathrm{Na}_{0.5} \mathrm{Bi}_{0.5} \mathrm{TiO}_{3}-x \% \mathrm{BaTiO}_{3}$ single crystals near the morphotropic phase boundary. Appl. Phys. Lett. 101, 141912 (2012).

27. Fujii, I. et al. Electric field induced lattice strain in pseudocubic $B i\left(\operatorname{Mg}_{1 / 2} \mathrm{Ti}_{1 / 2}\right)$ $\mathrm{O}_{3}$-modified $\mathrm{BaTiO}_{3}-\mathrm{BiFeO}_{3}$ piezoelectric ceramics. Appl. Phys. Lett. 108, 172903 (2016).

28. Endriss, A., Hammer, H., Hoffmann, M. J., Kolleck, A. \& Schneider, G. A. Microscopic and macroscopic ferroelectric-ferroelastic and piezoelectric behavior of PZT ceramics. J. Euro. Ceram. Soc. 19, 1229-1231 (1999).

29. Hall, D. A., Steuwer, A., Cherdhirunkorn, B., Mori, T. \& Withers, P. J. A high energy synchrotron $\mathrm{x}$-ray study of crystallographic texture and lattice strain in soft lead zirconate titanate ceramics. J. Appl. Phys. 96, 4245 (2004).

30. Paramanick, A., Damjanovic, D., Daniels, J. E., Nino, J. C. \& Jones, J. L. Origins of electro-mechanical coupling in polycrystalline ferroelectrics during subcoercive electrical loading. J. Am. Ceram. Soc. 94, 293-309 (2011).

31. Noheda, B. et al. Polarization rotation via a monoclinic phase in the piezoelectric $92 \% \mathrm{PbZn}_{1 / 3} \mathrm{Nb}_{2 / 3} \mathrm{O}_{3}-8 \% \mathrm{PbTiO}_{3}$. Phys. Rev. Lett. 86, 3891-3894 (2001).

32. Noheda, B. et al. Electric-field-induced phase transitions in rhombohedral $\mathrm{Pb}$ $\left(\mathrm{Zn}_{1 / 3} \mathrm{Nb}_{2 / 3}\right) 1-x \mathrm{TiO}_{3}$. Phys. Rev. B 65, 224101 (2002).

33. Tazaki, R., Fu, D., Itoh, M., Daimon, M. \& Koshihara, S. Lattice distortion under an electric field in $\mathrm{BaTiO}_{3}$ piezoelectric single crystal. J. Phys.: Condens. Matter 21, 215903 (2009).

34. Guo, R. et al. Origin of the high piezoelectric response in $\mathrm{PbZr}_{1-x} \mathrm{TixO}_{3}$. Phys. Rev. Lett. 84, 5423-5426 (2000).

35. Kitanaka, Y. et al. Non- $180^{\circ}$ polarization rotation of ferroelectric $\left(\mathrm{Bi}_{0.5} \mathrm{Na}_{0.5}\right)$ $\mathrm{TiO}_{3}$ single crystals under electric field. Phys. Rev. B 89, 104104 (2014).

36. Lalitha, K. V., Fancher, C. M., Jones, J. L. \& Ranjan, R. Field induced domain switching as the origin of anomalous lattice strain along non-polar direction in rhombohedral $\mathrm{BiScO}_{3}-\mathrm{PbTiO}_{3}$ close to the morphotropic phase boundary. Appl. Phys. Lett. 107, 052901 (2015).

37. Takata, M. The MEM/Rietveld method with nano-applications-accurate charge-density studies of nano-structure materials by synchrotron-radiation powder diffraction. Acta Cryst. A 26, 232-245 (2008).

38. Reszt, J.-T., Glazounov, A. E. \& Hoffmann, M. J. Analysis of intrinsic lattice deformation in PZT-ceramics of different compositions. J. Euro. Ceram. Soc. 21, 1349-1352 (2001).

39. Luo, C. et al. Hierarchical domain structure of lead-free piezoelectric $\left(\mathrm{Na}_{1 /}\right.$ $\left.{ }_{2} \mathrm{Bi}_{1 / 2}\right) \mathrm{TiO}_{3}-\left(\mathrm{K}_{1 / 2} \mathrm{Bi}_{1 / 2}\right) \mathrm{TiO}_{3}$ single crystals. J. Appl. Phys. 119, 174102 (2016).

40. Tanaka, H. et al. ENIGMA: maximum-entropy method program package for huge systems. J. Appl. Cryst. 35, 282-286 (2002).

41. Zalar, B., Lebar, A., Seliger, J. \& Blinc, R. NMR study of disorder in $\mathrm{BaTiO}_{3}$ and $\mathrm{SrTiO}_{3}$. Phys. Rev. B 71, 064107 (2005).

42. Levin, I., Krayzman, V. \& Woicik, J. C. Local structure in perovskite (Ba,Sr) $\mathrm{TiO}_{3}$ : reverse Monte Carlo refinements from multiple measurement techniques. Phys. Rev. B 89, 024106 (2014).

43. Zalar, B., Laguta, V. V. \& Blinc, R. NMR evidence for the coexistence of orderdisorder and displacive components in barium titanate. Phys. Rev. Lett. 90, 037601 (2003)

44. Ravel, B., Stern, E. A., Vedrinskii, R. I. \& Kraizman, V. Local structure and the phase transition of $\mathrm{BaTiO}_{3}$. Ferroelectrics 206, 407-430 (1998).

45. Levin, I., Krayzman, V. \& Woicik, J. C. Local-structure origins of the sustained Curie temperature in $(\mathrm{Ba}, \mathrm{Ca}) \mathrm{TiO}_{3}$ ferroelectrics. Appl. Phys. Lett. 102, 162906 (2013).

46. Singh, A., Moriyoshi, C., Kuroiwa, Y. \& Pandey, D. Visualization of $\mathrm{Bi}^{3+}$ offcentering in the average cubic structure of $\left(\mathrm{Ba}_{0.7} \mathrm{Bi}_{0.3}\right)\left(\mathrm{Ti}_{0.7} \mathrm{Fe}_{0.3}\right) \mathrm{O}_{3}$ at the electron density level. Appl. Phys. Lett. 103, 121907 (2013).

47. Singh, A., Moriyoshi, C., Kuroiwa, Y. \& Pandey, D. Evidence for diffuse ferroelectric phase transition and cooperative tricritical freezing of randomsite dipoles due to off-centered $\mathrm{Bi}^{3+}$ ions in the average cubic lattice of $\left(\mathrm{Ba}_{1}\right.$ $\left.{ }_{-x} \mathrm{Bi}_{x}\right)\left(\mathrm{Ti}_{1-x} \mathrm{Fe}_{x}\right) \mathrm{O}_{3}$. Phys. Rev. B 85, 064116 (2012).

48. Moriyoshi, C., Takeda, S., Kuroiwa, Y. \& Goto, M. Off-centering of a Bi ion in cubic phase of $\left(\mathrm{Bi}_{1 / 2} \mathrm{Na}_{1 / 2}\right) \mathrm{TiO}_{3}$. Jpn. J. Appl. Phys. 53, 09PD02 (2014).

49. Momma, K. \& Izumi, F. VESTA 3 for three-dimensional visualization of crystal, volumetric and morphology data. J. Appl. Cryst. 44, 1272-1276 (2011).

50. Wang, G. et al. Ultrahigh energy storage density lead-free multilayers by controlled electrical homogeneity. Energy Environ. Sci. 12, 582 (2019).

51. Wang, $\mathrm{D}$. et al. High energy storage density and large strain in $\mathrm{Bi}\left(\mathrm{Zn}_{2 / 3} \mathrm{Nb}_{1 / 3}\right)$ $\mathrm{O}_{3}$-doped $\mathrm{BiFeO}_{3}-\mathrm{BaTiO}_{3}$ ceramics. ACS Appl. Energy Mater 1, 4403 (2018).
52. Murakami, S. et al. High strain $(0.4 \%) \mathrm{Bi}\left(\mathrm{Mg}_{2 / 3} \mathrm{Nb}_{1 / 3}\right) \mathrm{O}_{3}-\mathrm{BaTiO}_{3}-\mathrm{BiFeO}_{3}$ lead-free piezoelectric ceramics and multilayers. J. Am. Ceram. Soc. 101 5428-5442 (2018)

53. Murakami, S. et al. Optimising dopants and properties in $\mathrm{BiMeO}_{3}(\mathrm{Me}=\mathrm{Al}$, $\mathrm{Ga}, \mathrm{Sc}, \mathrm{Y}, \mathrm{Mg}_{2 / 3} \mathrm{Nb}_{1 / 3}, \mathrm{Zn}_{2 / 3} \mathrm{Nb}_{1 / 3}, \mathrm{Zn}_{1 / 2} \mathrm{Ti}_{1 / 2}$ ) lead-free $\mathrm{BaTiO}_{3}-\mathrm{BiFeO}_{3}$ based ceramics for actuator applications. J. Euro. Ceram. Soc. 38, 4220-4231 (2018).

54. Levin, I., Laws, W. J., Wang, D. \& Reaney, I. M. Designing pseudocubic perovskites with enhanced nanoscale polarization. Appl. Phys. Lett. 111, 212902 (2017).

55. Aizawa, T., Fujii, I., Ueno, S., Suzuki, T. S. \& Wada, S. Effect of oxygen partial pressure during sintering on electric properties of $\mathrm{BiFeO}_{3}$-based piezoelectric ceramics. J. Ceram. Soc. Jpn. 127, 383-387 (2019).

56. Jones, L. et al. Optimizing multi-frame ADF-STEM for high-precision atomicresolution strain mapping. Ultramicrosc 179, 57-62 (2017).

57. Jones, L. \& Nellist, P. D. Identifying and correcting scan noise and drift in the scanning transmission electron microscope. Microsc. Microanal. 19, 1050-1060 (2013)

58. Sato, Y., Gondo, T., Miyazaki, H., Teranishi, R. \& Kaneko, K. Electron microscopy with high accuracy and precision at atomic resolution: in-situ observation of a dielectric crystal under electric field. Appl. Phys. Lett. 111, 062904 (2017).

\section{Acknowledgements}

The authors would like to thank Dr. S. Kawaguchi and Ms. N. Hikiji for their assistance during the experiments at SPring-8. The SXRD experiments were conducted with the approval of the Japan Synchrotron Radiation Research Institute (JASRI) (mainly proposal No. 2017A0074 and partially proposal No. 2018A0078). The STEM measurements were performed at the Ultramicroscopy Center, Kyushu University. This study was partially supported by JSPS KAKENHI, Grant-in-Aid for Scientific Research (B), 2017-2020 (JP17H02776, Y.K.), 2020-2023 (JP20H02641, Y.K.), and the MEXT Element Strategy Initiative to Form Core Research Center (JPMXP0112101001, S.W.).

\section{Author contributions}

The project was conceived and conducted by Y.K. and S.W.; S.K., I.F., S.U., and S.W. prepared the ceramic samples and measured the ferroelectric and piezoelectric responses Y.K., Y.N., and C.M. conducted the SXRD experiments. First, Y.N. analyzed the crysta structures to discuss with the authors the mechanism of piezoelectricity and ferroelectricity, and subsequently, Y.S. performed the STEM measurements. Y.K. and S.K. prepared the paper.

\section{Competing interests}

The authors declare no competing interests.

\section{Additional information}

Supplementary information is available for this paper at https://doi.org/10.1038/s43246020-00072-4.

Correspondence and requests for materials should be addressed to Y.K.

Reprints and permission information is available at http://www.nature.com/reprints

Publisher's note Springer Nature remains neutral with regard to jurisdictional claims in published maps and institutional affiliations.

Open Access This article is licensed under a Creative Commons Attribution 4.0 International License, which permits use, sharing, adaptation, distribution and reproduction in any medium or format, as long as you give appropriate credit to the original author(s) and the source, provide a link to the Creative Commons license, and indicate if changes were made. The images or other third party material in this article are included in the article's Creative Commons license, unless indicated otherwise in a credit line to the material. If material is not included in the article's Creative Commons license and your intended use is not permitted by statutory regulation or exceeds the permitted use, you will need to obtain permission directly from the copyright holder. To view a copy of this license, visit http://creativecommons.org/ licenses/by/4.0/

(C) The Author(s) 2020 